\title{
Scanning laser ophthalmoscopy of the optic disc at the level of the lamina cribrosa
}

\author{
Stefano Miglior, Luca Rossetti, Cristina Lonati and Nicola Orzalesi \\ Department of Ophthalmology, University of Milan, Institute of Biomedical Sciences, San Paolo Hospital, Milan, Italy
}

\begin{abstract}
A bstract
Purpose. To determine the suitability and reproducibility of optic disc morphometry performed on images focused at the level of the lamina cribrosa, obtained by means of the scanning laser ophthalmoscope (SLO).

Methods. Twenty-one eyes were imaged with argon blue and green, helium neon red and diode infrared laser sources of a scanning laser ophthalmoscope. Five images of the optic disc at the level of the lamina cribrosa were taken for each patient, digitized and traced by three trained observers, in order to identify the external contour of the optic disc and the inner edge of the neuroretinal rim. Dedicated software allowed the contours to be traced on the video and an estimate of the real sizes of optic disc parameters to be obtained according to Littman's equation. Standard deviation (SD) and coefficient of variation $(\mathrm{CV})$ were used to determine the intra- and interobserver reproducibility in measuring disc, dark annulus (DA), and reflective center (RC) areas, DA area/disc area (DA/D) and $\mathrm{RC}$ area/disc area (RC/D) ratios of each set of images taken with all laser wavelengths.
\end{abstract}

Results. A high contrast between the dark annulus of the lamina and the central highly reflective area was achieved at all laser wavelengths. The ranges of CVs for all of the observers, using all the laser wavelengths, were as follows: disc: 0.018 0.036; dark annulus: 0.015-0.039; reflective center: 0.0140.031; DA/D: $0.005-0.01$; RC/D: 0.007-0.018. No significant difference was observed between the measurements performed on the images acquired, using the different laser wavelengths.

Conclusions. SLO imaging of the optic disc at the level of the lamina cribrosa proved to be suitable and highly reproducible at all laser wavelengths. The coefficients of variation of the measurements of optic disc parameters obtained using this technique are smaller than those obtained by means of con-

Correspondence: Dr. Stefano Miglior, Clinica Oculistica, Universita' degli Studi di Milano, Istituto di Scienze Biomediche, Ospedale S. Paolo, via di Rudini’ 8, 20142 Milano, Italy; E-mail: smi@imiucca.csi.unimi.it ventional photographic methods. Curr. Eye Res. 17: 453-461, 1998.

Key words: scanning laser ophthalmoscope; optic disc; lamina cribrosa; glaucoma; reproducibility

\section{Introduction}

A reproducible documentation of the normal and glaucomatous characteristics of the optic nerve may be obtained either by means of manual planimetry or automated systems.

However, the advantages associated with the measurements of the optic disc parameters are hampered by the problems related to the accurate identification of the edge of the cup. This is particularly difficult in the case of cups with temporal flat slopes when using manual planimetry (1-3). The identification of cup edge may be obtained by means of photographicbased systems such as stereophotogrammetry (4) and flicker analysis (5), which allow assessment of the changes in the slope of the cup wall. The identification of cup margin in automated systems that permit three dimensional reconstructions of the disc, is obtained by means of arbitrary definitions such as "the first point along the radial profile that is $150 \mu \mathrm{m}$ below the level of the disc edge on that particular profile" (6), and is the standard definition of the optic nerve head analyzer (ONHA). In the case of confocal laser systems, the measurements of disc parameters are related to the contour of the disc margin or to a ring that is automatically superimposed on the imaged parapapillary region (7-14). With these systems, the definition of cup margin depends upon the localization of the "reference plane."

Imaging of the disc, focused at the level of the lamina cribrosa (the sharpest visualization of the lamina cribrosa pores), may help in better identifying the border between the portion of the lamina which is perpendicularly crossed by the ganglion cell axons and that portion which is not crossed or where nerve fibers run parallel to the surface of the lamina. Imaging of the lamina cribrosa has been successfully obtained by means of an 
experimental scanning laser ophthalmoscope (15) and may be clinically performed by means of the Rodenstock scanning laser ophthalmoscope (SLO) (16-18), which enables highcontrast images and a "virtual" tomography of the fundus to be obtained using different laser sources $(19,20)$.

The aim of this study was to give a detailed description of optic disc imaging at the level of the lamina cribrosa, by means of argon blue (488 $\mathrm{nm})$ and green $(514 \mathrm{~nm})$, helium neon red $(633 \mathrm{~nm})$ and diode infrared $(780 \mathrm{~nm})$ scanning laser ophthalmoscopy in normal and glaucomatous eyes, and to assess the intra-observer and inter-observer variability of optic disc morphometry performed on SLO images obtained at four different laser wavelengths.

\section{Subjects and methods}

Eleven subjects ( 7 females and 4 males, age range 32-74 yrs), for a total of 21 eyes, gave their informed consent to be enrolled in the study, which was performed according to the principles of the Declaration of Helsinki. The sample included 8 eyes affected by primary open angle glaucoma (POAG) at different stages of the disease and eyes with a wide range of disc, rim and cup sizes, and different morphological characteristics (cups with temporal flat slopes; steep "punched-out" cups; peripapillary atrophy). The eyes with a history of POAG were pooled together with normal eyes in order to have a group of eyes with completely different morphological features, such as very narrow or very thick neuroretinal rims, enlarged cups or absence of cups, etc., which may affect the evaluation of the disc, particularly the assessment of the edge between the rim and the cup, on a two-dimensional SLO image. Tilted myopic optic discs were not included in the study.

All of the patients underwent keratometry (Javal-Schiotz), refraction examination and axial length measurement (standard A-scan ultrasonography), followed by scanning laser ophthalmoscopy and standard optic disc photography. Scanning laser examination can be routinely performed on undilated pupils. However, a mydriasis of about $4-5 \mathrm{~mm}$ was preferred in order to avoid pupillary fluctuation, which makes the adjustment of the laser beam on the retinal and disc surfaces more difficult and time consuming. Intraocular pressure (IOP, by Goldmann applanation tonometry) was measured immediately after all these procedures in order to have the closest estimate of IOP during ophthalmoscopy. The IOP of all of the patients ranged from 15 to $20 \mathrm{~mm} \mathrm{Hg}$.

A scanning laser ophthalmoscope (101 Rodenstock, Ottobrun, Germany) was used, and ophthalmoscopy was performed by means of an argon laser (488 $\mathrm{nm}$ blue and $514 \mathrm{~nm}$ green), a helium neon laser (633 nm red) and a diode laser (780 nm infrared). Laser intensities ranged from 140 to $290 \mu \mathrm{W}$. A confocal aperture of $2 \mathrm{~mm}$, a field angle of $20^{\circ}$ and a preset video gain on the automatic position were used in all cases. An accurate setting of refraction values and an accurate focusing of the laser beam on the corneal surface were attained before imaging.

Fundus imaging was centered on the optic disc with the aid of a fixation spot, which helps the examined eye to remain still in the correct position during the examination. Initial focusing on optic disc surface was followed by the backward focusing on the lamina cribrosa, which was reached when the pores of the lamina became sharp. In eyes with no cup, a backward focusing of about 2-3 dptrs (which was the average backward focusing needed for our cases) was used. In such cups, the pores of the lamina cribrosa were not detectable.

All of the images were directly grabbed and digitized (Targa+ 16, True Vision) with a resolution of $511 \times 476$ pixels on to the Eyelab system (Digigraphic Technologies, Treviso, Italy). Optic disc morphometry was performed on the digitized video image by means of dedicated software, which takes into account refraction and keratometry, in order to obtain an estimate of the real size of the objects on the fundus of the eye according to Littman's equations (21). The software was developed by Digigraphic Technologies in collaboration with one of the Authors (S. M.). Estimation of the field-size and enlargement factor needed for any SLO instrument were calculated, using simple linear measurements, and applied to the software.

Definition of disc and cup borders was not based on contour evaluation, but on the contrast between the peripheral dark annulus and the bright central area, which is highly enhanced at the level of the lamina cribrosa at all laser wavelengths. Throughout the manuscript, the terms "dark annulus" (DA) and "reflective center" (RC) refer to what might be interpreted as the rim and the cup, respectively.

Disc border was defined as the boundary line between the dark annulus and the area surrounding the disc (lighter than the annulus). The border of the reflective center was defined as the boundary line between the dark annulus and the central, highly reflective portion of the lamina cribrosa. In the seven cases where the edge of the reflective center was not clearly defined (e.g. Figs. 1 and 4), its contour included all the detectable pores of the lamina cribrosa. If a vessel (dark) unattached to the dark annulus was found within the reflective center, it was recorded as a part of the reflective center and not as a part of the dark annulus. The boundaries were marked with a continuous line traced on the video by means of a mouse. The software automatically calculated the number of pixels within the traced contours and provided the transformation in actual measurements, according to the above mentioned methods. The following parameters were then calculated: disc area, dark annulus area, reflective center area, DA area/disc area ratio (DA/D), and $\mathrm{RC}$ area/disc area ratio (RC/D).

To evaluate the reproducibility of the technique, scanning laser ophthalmoscopy was repeated on five different days. Each day three photographs were taken, and the best one (decision based on observers' agreement) was chosen for the analysis.

To evaluate the agreement between the measurements performed on SLO and standard photographic images of the optic disc, the absolute measurements of the optic disc images obtained by the SLO and analyzed by observer 2 (C. L.) were compared with photographic optic disc measurements performed by the same observer. For this purpose, optic disc photography and morphometry were performed as previously described (22-25). The true amplitude of the planimetric optic disc area 
was calculated using Littman's equation, which takes into account ocular axial length and keratometry.

The intra-observer "intra-image" variability of the SLO measurements of three independent observers was calculated as the standard deviation (SD) (average of 21 cases) and the coefficient of variation $(\mathrm{CV})$ of five repeated measurements of the same image for each type of laser wavelength. The intraobserver "inter-image" variability of the SLO measurements of three independent observers was calculated as the SD (average of 21 cases) and the $\mathrm{CV}$ of a single measurement of five different images of the same optic nerve head for each type of laser wavelength.

The inter-observer "intra-image" variability was calculated as the SD (average of 21 cases) and the CV of the five measurements of the same image performed by the three observers (for a total of 15 measurements), for each type of laser wavelength. The inter-observers "inter-image" variability was calculated as the SD (average of 21 cases) and CV of a single measurement of five different images of the same optic nerve head performed by the three observers (for a total of 15 measurements), for each type of laser wavelength.

SD and CV (standard deviation divided by the mean) were calculated according to standard procedures (26). Nonparametric Wilcoxon and Kruskall-Wallis tests were used to compare
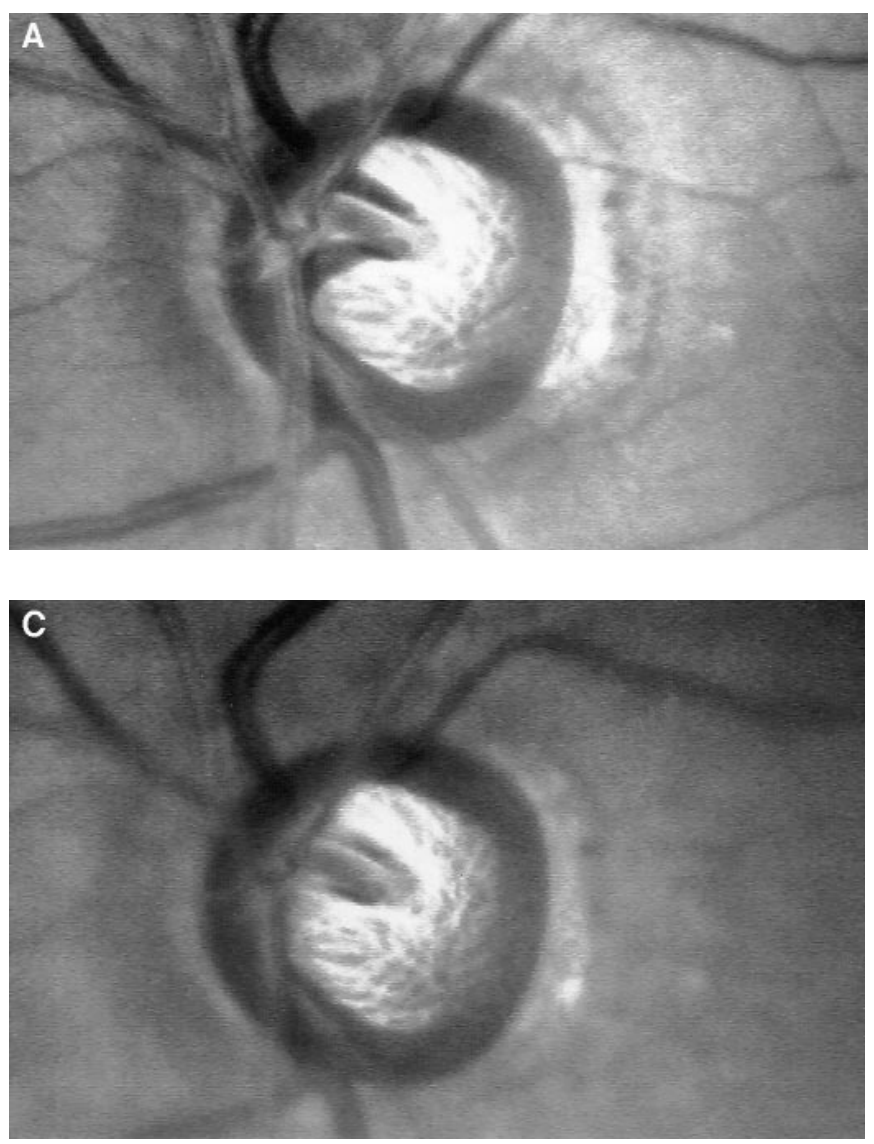

optic disc measurements among groups. Regression statistics (linear regression) were used to assess relationships between the SDs and CVs ("y", dependent variables in the model) of the measurements of the optic disc parameters and their sizes ("x", independent variables of the model). All calculations were performed with SPSS (version 6.1 for Macintosh) software.

\section{Results}

Optic disc visualization was extremely sharp at all laser wavelengths, even with a power lower than $180 \mu \mathrm{W}$ (Fig. 1). In all cases and using all laser wavelengths, the image of the fundus (nerve fiber bundles, superficial large vessels) tended to blur when backward focusing. On average, a backward focusing of 2.5 dptrs was needed to focus the lamina cribrosa, which appeared to be highly reflective white or light grey, allowing identification of the pores in all cases. The dark annulus was always much darker than the central portion of the lamina and darker than the surrounding tissues. In correlation to the dark annulus, the pores were not visible. These features were particularly clear using the infrared laser (Fig. 1, bottom right). Regions of peripapillary atrophy were always whitish, perfectly distinguishable from the dark annulus. The veins appeared as dark as the annulus, and sometimes darker, where-
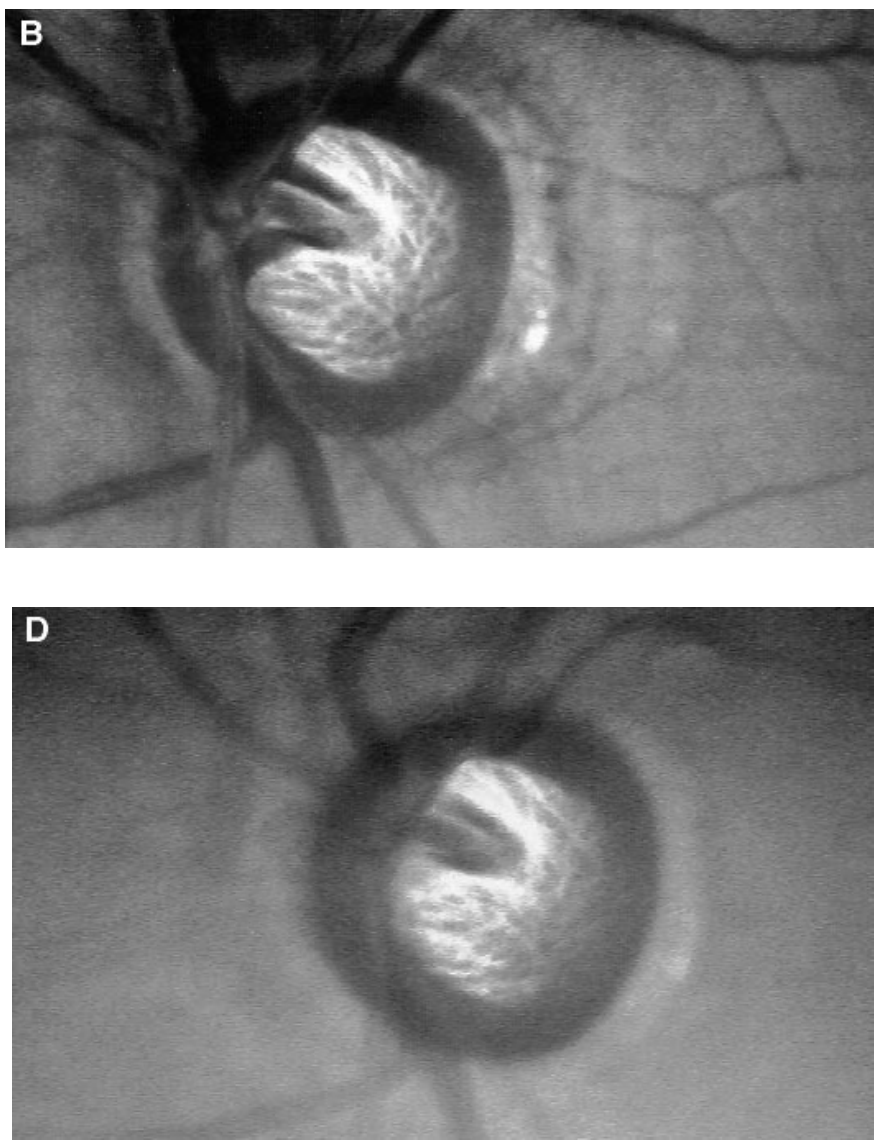

Figure 1. (A) A rgon blue $(488 \mathrm{~nm}$ ). (B) A rgon green ( $514 \mathrm{~nm}$ ). (C) Helium neon red ( $633 \mathrm{~nm})$. (D) D iode infrared ( $780 \mathrm{~nm}$ ). A II images are obtained with confocal $2 \mathrm{~mm}$ and $20^{\circ}$-field angle. The lamina cribrosa appears white or light greyish, allowing the pores to be identified at all laser wavelengths. The dark annulus is much darker than the surrounding tissues and is detectable at all laser wavelengths. 

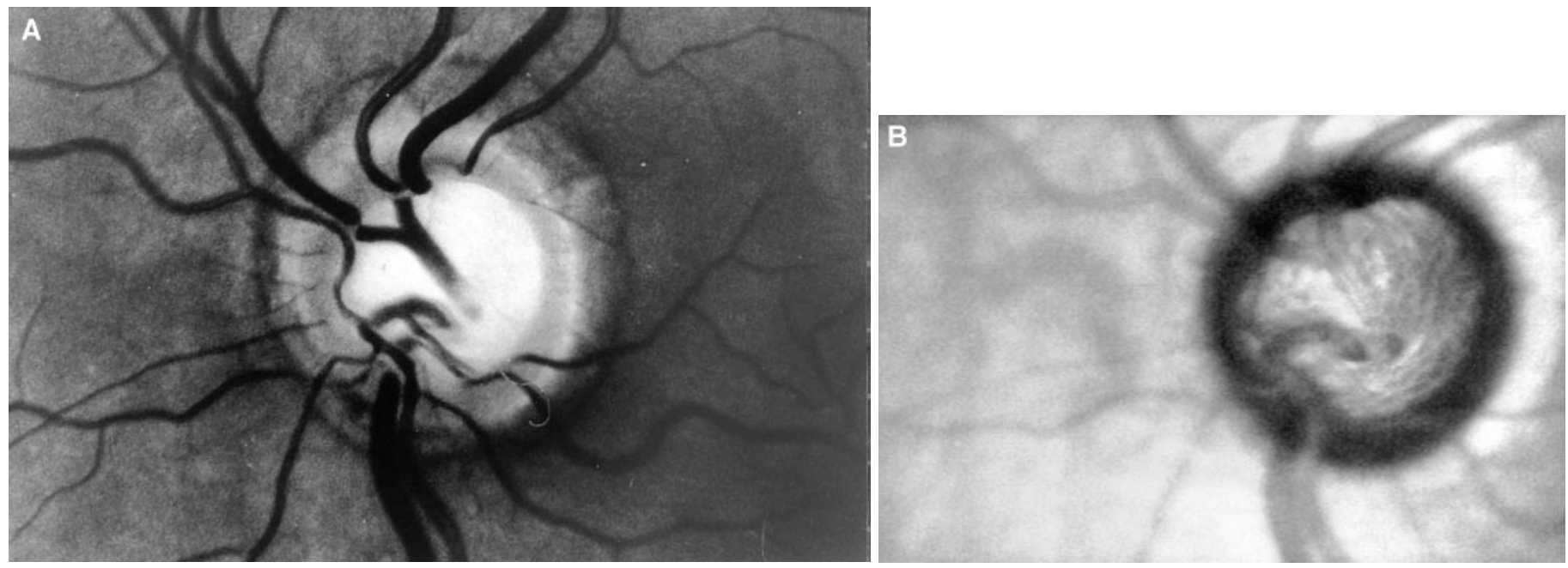

Figure 2. (A) Black and white photograph of optic disc with "punched out" cup. (B) Infrared image of the same optic disc (20 -field angle, confocal $2 \mathrm{~mm}$ ). I $\mathrm{n}$ both cases the edge between the reflective center and the dark annulus is easily detectable.

as the arteries appeared lighter than the annulus at all laser wavelengths. It was difficult to identify the exact border between the vessel and the dark annulus when a vessel was attached to the annulus itself.

The optic disc size estimates were in good agreement with the measurements performed on conventional stereophotographs (Figs. 2, 3,4) by means of computer-assisted manual morphometry (Table 1), the small differences between their means being statistically not significant in all cases (Table 2 ).

The measurements of the optic disc parameters performed by the three observers were consistent at each laser wavelength, and a constant tendency to obtain progressively increasing optic disc size estimates from the red to the infrared laser, as well as dark annulus size estimates from the blue to the infrared laser, was in fact observed in the measurements of all three observers (Table 3); the difference in disc measurements was statistically significant for all of the three observers $(\mathrm{p}=$ 0.01, Kruskal-Wallis test).

Low intra-observer "intra-image" and "inter-image" variabilities were found for the three independent observers in the measurement of optic disc parameters. DA/D and RC/D showed the lowest mean SDs and CVs (Table 4). In some cases, it was possible to obtain CVs smaller than 0.001 or larger than 0.1 for both DA/D and RC/D (Table 5) as the result of the significant associations between the CVs of both DA/D and RC/D with DA area, RC area, DA/D and RC/D (Table 6). The larger the DA area and the DA/D, the smaller the $\mathrm{CV}$ of DA/D measurement. Conversely, the smaller the RC area and the RC/D, the larger the $\mathrm{CV}$ of $\mathrm{RC} / \mathrm{D}$ measurement. Such associations were consistent among all three observers. Low inter-observer "intra-
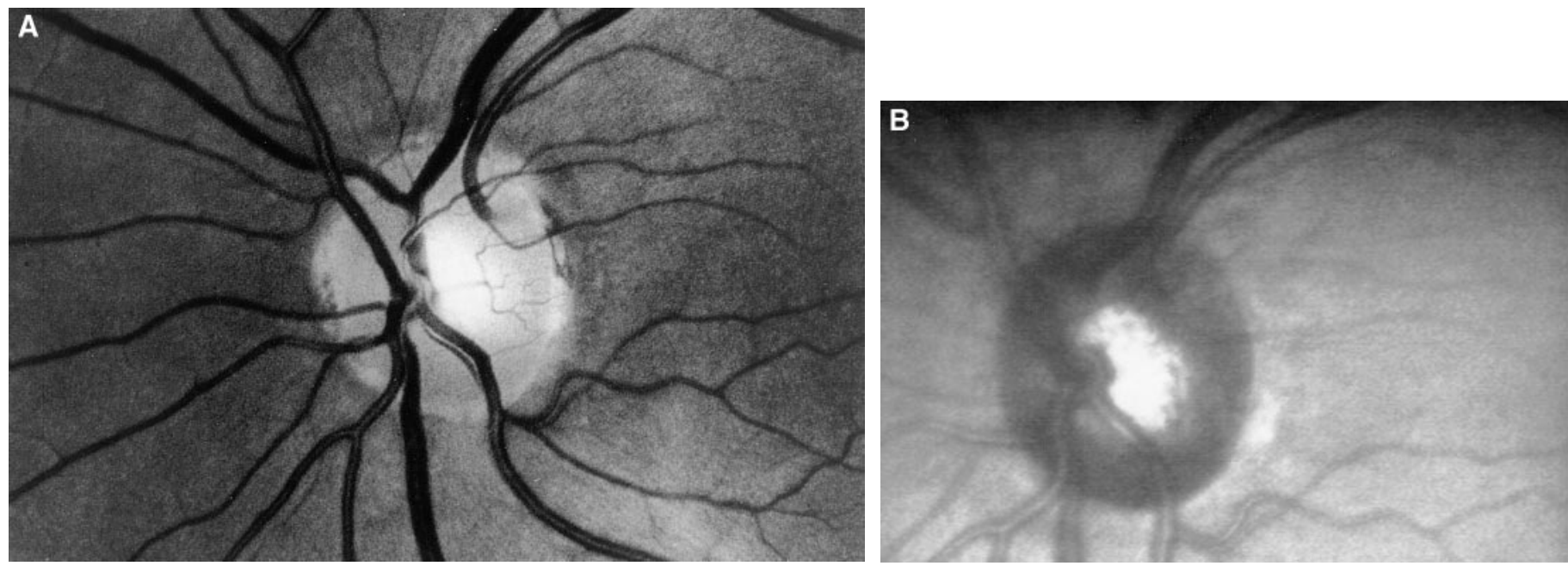

Figure 3. (A) Black and white photograph of optic disc with temporal flat slope of the cup. (B) Infrared image of the same optic disc (20field angle, confocal $2 \mathrm{~mm}$ ). SLO image of the optic disc, focused at the level of the lamina cribrosa, allows the border of the reflective center to be easily identified. 

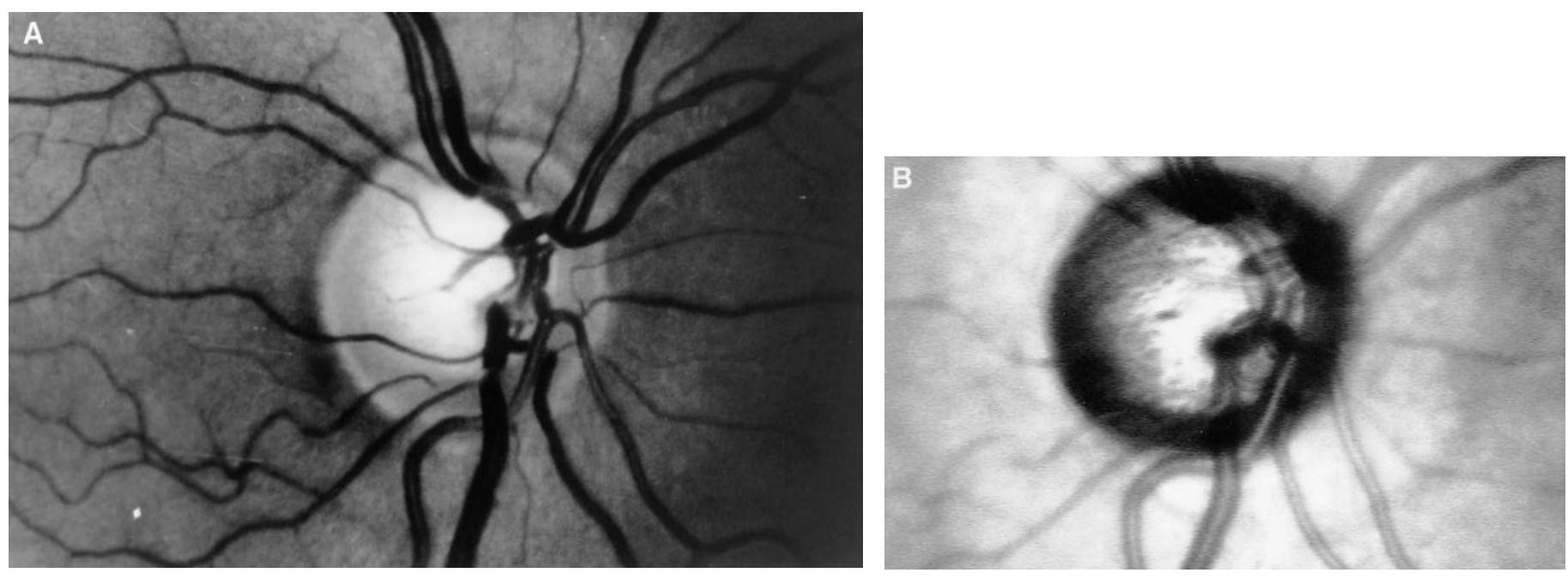

Figure 4. (A) Black and white photograph of optic disc with temporal flat slope of the cup. (B) Infrared image of the same optic disc (20field angle, confocal $2 \mathrm{~mm}$ ). A Iso in this case, SLO image of the optic disc, focused at the level of the lamina cribrosa, allows the border of the reflective center to be easily identified.

image" and "inter-image" variabilities were found in the measurement of optic disc parameters, particularly for disc, DA and DA/D (Table 7).

\section{Discussion}

This study attempts to give a detailed description of twodimensional optic disc imaging at the level of the lamina cribrosa by means of argon blue and green, helium neon red and diode infrared scanning laser ophthalmoscopy, and to evaluate the intra- and inter-observer reproducibility of the measurements of optic disc parameters. Our results indicate that imaging of the optic nerve head at the level of the lamina cribrosa by means of scanning laser ophthalmoscopy is feasible, and that optic disc measurements performed by independent observers are repeatable, reproducible and in good agreement with planimetric measurements performed on standard optic disc photographs.

Images of the human lamina cribrosa have already been successfully obtained by means of a confocal scanning laser ophthalmoscope in order to look for regional differences in its structure, and particularly for morphometric analysis of the pores (15-18). This is possible, as confocal scanning laser ophthalmoscopy allows a virtual tomography of the fundus of the eye to be performed: light reflected from focused surfaces passes by and contributes to image construction, whereas scattered light arising from defocused tissues or from points lying off the optical axis of the system is excluded. Moreover, it has been shown that a series of images of the same optic disc may be aligned to compensate for eye movements by a combination of automatic and interactive processing in order to reject artifacts and reduce noise by image averaging, thus improving the quality of the images $(17,18)$.

The SLO image of the optic disc focused at the level of the lamina cribrosa is characterized by a dark annulus and a bright central area. Of particular importance is whether the images correspond to known structures of the optic disc.
One hypothesis is that the dark annulus and the highly reflective area at the center of the disc correspond to regions of the lamina which are crossed or not crossed by the nerve fibers. In fact, it is very likely that the dark band is due to the channel-

Table 1. Optic disc sizes $\left(\mathrm{mm}^{2}\right)$ calculated on conventional photographs and on the video images obtained by means of the scanning laser ophthalmoscope (Obs. 2, 21 cases, mean of five repeated measurements)

\begin{tabular}{llllll}
\hline Case no. & Photo* & Blue $^{\dagger}$ & Green $^{\dagger}$ & Red $^{\dagger}$ & Infrared $^{\dagger}$ \\
\hline 1 & 3.216 & 3.286 & 3.426 & 3.151 & 3.157 \\
2 & 3.101 & 2.959 & 2.938 & 2.99 & 3.208 \\
3 & 2.53 & 2.367 & 2.369 & 2.299 & 2.407 \\
4 & 2.213 & 2.242 & 2.271 & 2.225 & 2.236 \\
5 & 2.73 & 2.518 & 2.527 & 2.517 & 2.537 \\
6 & 2.448 & 2.585 & 2.511 & 2.5 & 2.548 \\
7 & 2.408 & 2.195 & 2.165 & 2.069 & 2.086 \\
8 & 2.569 & 2.886 & 2.838 & 2.941 & 2.742 \\
9 & 2.579 & 2.782 & 2.807 & 2.752 & 2.866 \\
10 & 2.61 & 2.091 & 2.114 & 2.118 & 2.176 \\
11 & 3.25 & 3.424 & 3.398 & 3.376 & 3.523 \\
12 & 2.278 & 2.523 & 2.501 & 2.438 & 2.494 \\
13 & 2.521 & 2.632 & 2.582 & 2.569 & 2.552 \\
14 & 3.53 & 3.499 & 3.275 & 3.211 & 3.308 \\
15 & 2.65 & 2.735 & 2.708 & 2.821 & 3.051 \\
16 & 2.405 & 2.743 & 2.799 & 2.589 & 2.804 \\
17 & 2.358 & 2.436 & 2.514 & 2.394 & 2.616 \\
18 & 3.105 & 3.318 & 3.288 & 3.299 & 3.146 \\
19 & 3.124 & 3.195 & 3.162 & 3.277 & 3.254 \\
20 & 2.732 & 2.899 & 2.763 & 2.76 & 2.748 \\
21 & 3.183 & 3.208 & 3.326 & 3.152 & 3.429 \\
\hline
\end{tabular}

*Black \& white pictures; "black and white video images obtained by means of blue argon $(488 \mathrm{~nm})$, green $\operatorname{argon}(514 \mathrm{~nm})$, red helium neon $(633 \mathrm{~nm})$ and infrared diode $(780 \mathrm{~nm})$. 
Table 2. Average measurements (SD) of optic disc parameters performed on conventional photographs and on the video images obtained by means of all of the laser wavelengths of the scanning laser ophthalmoscope (observer 2, 21 cases)

\begin{tabular}{lccccc}
\hline Area of photo & Photo* & Blue & Green & Red $^{\#}$ & Infrared $^{\#}$ \\
\hline Disc area & $2.74(0.38)$ & $2.79(0.42)$ & $2.77(0.41)$ & $2.74(0.41)$ & $2.80(0.42)$ \\
DA area & $1.86(0.61)$ & $1.81(0.67)$ & $1.82(0.65)$ & $1.83(0.63)$ & $1.87(0.62)$ \\
RC area & $0.92(0.68)$ & $0.97(0.76)$ & $0.95(0.74)$ & $0.94(0.67)$ & $0.93(0.74)$ \\
DA/D & $0.68(0.24)$ & $0.65(0.25)$ & $0.66(0.25)$ & $0.67(0.24)$ & $0.67(0.24)$ \\
RC/D & $0.32(0.24)$ & $0.34(0.25)$ & $0.34(0.25)$ & $0.33(0.24)$ & $0.32(0.24)$ \\
\hline
\end{tabular}

*Black and white pictures; \#SLO video images are obtained by means of blue argon (488 nm), green argon (514 nm), red helium neon (633) and infrared diode (780). Disc, DA and RC areas are presented in $\mathrm{mm}^{2}$. None of the differences between SLO measurements and photographic measurements resulted to be statistically significant at the level of 0.05 (Wilcoxon test).

ling of the light into the tissue with no significant return, as the fibers may act as fiberoptics parallel to the light. On the other hand, the white central portion of the disc, where the pores are detectable, may correspond to the bared portion of the lamina itself (i.e. the bottom of the cup), which indeed is highly reflective. However, it is also likely that the highly reflective image of this region be related to a different direction of nerve fibers (running parallel to the surface of the lamina and transverse to the light), thus losing their optical fiber effect. As a consequence the light impinges on this surface and is scattered backward thus giving a bright reflection. If this is the case, the resulting images of these portions of the lamina and particularly the border between the dark annulus and the reflective center would heavily depend on the orientation of the fibers and not necessarily on their presence.

Other possible interpretations are the following: (1) the central reflective area may be related to the function of the depth of the cup; (2) the dark annulus area may be a reflection of more blood supply in the neuroretinal rim, whereas the central reflective area may indicate a lack of blood supply in the depth of the cup.

Our results indicate that the estimate of the size of optic disc structures is quite similar when using blue, green and red lights and is slightly larger when using the infrared light, although the images acquired with different wavelengths show some differences, particularly in peripapillary atrophy. This further supports the hypothesis that SLO image of the optic disc is independent of wavelength, indicating that the different optical behaviour of the dark annulus and the reflective center is likely one of direction. The fact that disc and dark annulus sizes are slightly larger when using infrared light could be explained by the higher penetration of infrared light through superficial fundus tissues, allowing light radiation to pass through the thin spur of the scleral ring and be reflected from the most peripheral ring of the lamina cribrosa, which is usually hidden when ophthalmoscopy is performed using visible wavelengths.

The measurements of optic disc parameters with SLO were in good agreement with planimetric measurements performed on the same eyes using a conventional photographic method (22-25). The values obtained with the two techniques were comparable although different criteria were used to define the "cup" and the "rim." A real test of accuracy would require the direct SLO examination of cadaver eyes, or an eye model. However, our planimetric measurements, performed on redfree photographs, using dedicated software, have been validated by a comparison with measurements performed on cadaver human eyes (24).

Table 3. Average measurements (SD) of optic disc parameters by means of the four different laser sources

\begin{tabular}{ccccc}
\hline & Blue & Green & Red & Infrared \\
\hline Obs 1 & & & & \\
Disc & $2.78(0.41)$ & $2.82(0.46)$ & $2.77(0.43)$ & $2.84(0.43)$ \\
DA & $1.74(0.65)$ & $1.78(0.64)$ & $1.77(0.62)$ & $1.81(0.64)$ \\
RC & $1.05(0.80)$ & $1.03(0.80)$ & $1.00(0.81)$ & $1.03(0.83)$ \\
DA/D & $0.64(0.27)$ & $0.65(0.26)$ & $0.66(0.26)$ & $0.66(0.26)$ \\
RC/D & $0.36(0.27)$ & $0.35(0.26)$ & $0.34(0.26)$ & $0.34(0.26)$ \\
Obs 2 & & & & \\
Disc & $2.79(0.42)$ & $2.77(0.41)$ & $2.74(0.41)$ & $2.80(0.42)$ \\
DA & $1.81(0.67)$ & $1.82(0.65)$ & $1.83(0.63)$ & $1.87(0.62)$ \\
RC & $0.97(0.76)$ & $0.95(0.74)$ & $0.94(0.67)$ & $0.93(0.74)$ \\
DA/D & $0.65(0.25)$ & $0.66(0.25)$ & $0.67(0.24)$ & $0.67(0.24)$ \\
RC/D & $0.34(0.25)$ & $0.34(0.25)$ & $0.33(0.24)$ & $0.32(0.24)$ \\
Obs 3 & & & & \\
Disc & $2.78(0.39)$ & $2.78(0.40)$ & $2.76(0.39)$ & $2.82(0.40)$ \\
DA & $1.77(0.68)$ & $1.78(0.67)$ & $1.79(0.67)$ & $1.81(0.66)$ \\
RC & $1.01(0.79)$ & $1.00(0.81)$ & $0.97(0.79)$ & $1.01(0.82)$ \\
DA/D & $0.65(0.27)$ & $0.65(0.27)$ & $0.66(0.26)$ & $0.66(0.26)$ \\
RC/D & $0.35(0.27)$ & $0.34(0.27)$ & $0.34(0.26)$ & $0.34(0.26)$ \\
Overall & & & & \\
Disc & $2.78(0.41)$ & $2.79(0.42)$ & $2.75(0.41)$ & $2.82(0.42)$ \\
DA & $1.77(0.66)$ & $1.79(0.65)$ & $1.79(0.64)$ & $1.83(0.64)$ \\
RC & $1.01(0.78)$ & $0.99(0.77)$ & $0.95(0.74)$ & $0.98(0.79)$ \\
DA/D & $0.64(0.26)$ & $0.65(0.26)$ & $0.66(0.25)$ & $0.66(0.25)$ \\
RC/D & $0.35(0.26)$ & $0.34(0.26)$ & $0.33(0.25)$ & $0.33(0.25)$ \\
\hline
\end{tabular}

DA: dark annulus; RC: reflective center; DA/D: dark annulus area/disc area ratio; RC/D: reflective center area/disc area ratio. Disc, DA and $\mathrm{RC}$ are in $\mathrm{mm}^{2}$. 
Table 4. Intra-observer "intra-image variability" for infrared images: standard deviation, average of the 21 cases. Coefficient of variation is reported in parenthesis. Results obtained with the other laser wavelengths are not reported in the Table as they are very similar to those obtained with infrared light.

\begin{tabular}{lccc}
\hline & Obs. 1 & Obs. 2 & Obs. 3 \\
\hline Disc & $0.044(0.016)$ & $0.028(0.010)$ & $0.025(0.009)$ \\
DA & $0.032(0.018)$ & $0.024(0.015)$ & $0.018(0.011)$ \\
RC & $0.024(0.036)$ & $0.021(0.036)$ & $0.015(0.025)$ \\
DA/D & $0.006(0.011)$ & $0.005(0.011)$ & $0.004(0.007)$ \\
RC/D & $0.005(0.029)$ & $0.005(0.034)$ & $0.004(0.020)$ \\
\hline
\end{tabular}

Intra-observer "inter-image variability" for infrared images: standard deviation, average of the 21 cases. Coefficient of variation is reported in parenthesis. Results obtained with the other laser wavelengths are not reported in the Table as they are very similar to those obtained with infrared light.

\begin{tabular}{lccc}
\hline & Obs. 1 & Obs. 2 & Obs. 3 \\
\hline Disc & $0.041(0.015)$ & $0.037(0.013)$ & $0.030(0.011)$ \\
DA & $0.030(0.017)$ & $0.027(0.018)$ & $0.020(0.012)$ \\
RC & $0.020(0.033)$ & $0.026(0.035)$ & $0.017(0.026)$ \\
DA/D & $0.004(0.008)$ & $0.006(0.013)$ & $0.004(0.008)$ \\
RC/D & $0.004(0.027)$ & $0.006(0.030)$ & $0.004(0.021)$ \\
\hline
\end{tabular}

Disc $\left(\mathrm{mm}^{2}\right)$; DA: dark annulus $\left(\mathrm{mm}^{2}\right)$; RC: reflective center $\left(\mathrm{mm}^{2}\right)$; DA/D: dark annulus area/disc area ratio; RC/D: reflective center area/disc area ratio.

The SLO image of the optic disc, focused at the level of the lamina cribrosa, yields a dark annulus with a contrast which surpasses that of conventional fundus photography. Assessment of the edge of the reflective center is then obtained by evaluating image contrasts and not by evaluating contours, which allowed the border line between the two portions of the lamina to be identified in all of the eyes investigated. In our study, the same holds for the identification of disc border, which was clearly distinguishable from the surrounding tissues, for all laser sources. As a consequence of this, the identification of the edge of the reflective center in cups with temporal flat slopes (a condition which frequently hampers the evaluation of conventional optic disc photographs (1-3)) may be more easily achieved (Figs. 3 and 4). This is reflected by the substantial improvement in CVs of the measurement of optic disc parameters obtained in our study (range 0.008-0.035), with respect to those obtained using conventional photography and manual planimetry (range 0.02-0.04) $(24,25)$.

The similar intra-observer "intra-image" and "inter-image" variabilities reported by the three observers indicate that the variability of the measurements does not depend on variations in image acquisition at different times, but arises from the variability in identifying and tracing the outlines of disc parameters on the video (pure observer variability). This is consistent with the values of inter-observer variability, which are higher than those obtained for intra-observer variability. Actually, the
Table 5. Intra-observer "intra-image variability" for infrared images: ranges of SD and CV of five repeated measurements of the same image of each of the 21 cases.

\begin{tabular}{llrrr}
\hline & & \multicolumn{1}{c}{ Obs. 1 } & \multicolumn{1}{c}{ Obs. 2 } & \multicolumn{1}{c}{ Obs. 3 } \\
\hline Disc & (SD) & $0.015-0.081$ & $0.006-0.067$ & $0.006-0.081$ \\
& $(\mathrm{CV})$ & $0.001-0.025$ & $0.006-0.031$ & $0.001-0.031$ \\
DA & (SD) & $0.011-0.064$ & $0.01-0.054$ & $0.007-0.064$ \\
& (CV) & $0.005-0.045$ & $0.006-0.051$ & $0.002-0.028$ \\
RC & $(\mathrm{SD})$ & $0.003-0.072$ & $0.005-0.057$ & $0.002-0.058$ \\
& $(\mathrm{CV})$ & $0.007-0.194$ & $0.011-0.103$ & $0.004-0.062$ \\
DA/D & (SD) & $0.0008-0.016$ & $0.002-0.011$ & $0.0007-0.01$ \\
& $(\mathrm{CV})$ & $0.001-0.042$ & $0.001-0.035$ & $0.001-0.020$ \\
RC/D & $(\mathrm{SD})$ & $0.0009-0.011$ & $0.002-0.011$ & $0.0006-0.01$ \\
& $(\mathrm{CV})$ & $0.004-0.199$ & $0.006-0.113$ & $0.003-0.045$ \\
\hline
\end{tabular}

Intra-observer "inter-image variability": ranges of SD and CV of a single measurement of five different images of 21 cases

\begin{tabular}{llrrr}
\hline & & \multicolumn{1}{c}{ Obs. 1} & \multicolumn{1}{c}{ Obs. 2 } & \multicolumn{1}{c}{ Obs. 3 } \\
\hline Disc & (SD) & \multicolumn{1}{c}{$0.018-0.07$} & $0.008-0.099$ & $0.01-0.081$ \\
& (CV) & $0.002-0.033$ & $0.007-0.025$ & $0.004-0.031$ \\
DA & (SD) & $0.011-0.064$ & $0.008-0.053$ & $0.006-0.064$ \\
& (CV) & $0.006-0.062$ & $0.007-0.032$ & $0.003-0.029$ \\
RC & (SD) & $0.002-0.053$ & $0.005-0.099$ & $0.0008-0.058$ \\
& $(\mathrm{CV})$ & $0.007-0.100$ & $0.011-0.149$ & $0.006-0.063$ \\
DA/D & $(\mathrm{SD})$ & $0.0004-0.016$ & $0.002-0.018$ & $0.0005-0.010$ \\
& $(\mathrm{CV})$ & $0.002-0.052$ & $0.0005-0.028$ & $0.0006-0.023$ \\
RC/D & $(\mathrm{SD})$ & $0.0004-0.011$ & $0.002-0.018$ & $0.0003-0.010$ \\
& (CV) & $0.007-0.105$ & $0.003-0.148$ & $0.004-0.058$ \\
\hline
\end{tabular}

DA: dark annulus; RC: reflective center; DA/D: dark annulus area/disc area ratio; RC/D: reflective center area/disc area ratio. SDs of Disc, DA and RC are in $\mathrm{mm}^{2}$. Results obtained with the other laser wavelengths are not reported in the Table as they are very similar to those obtained with infrared light.

precise setting of the instrument allows substantially identical images of optic disc structures to be obtained at different times.

As far as the intra-image variability is concerned, the CVs of relative measurements (DA/D and RC/D) were smaller than the CVs of absolute measurements (disc, DA and RC areas), this being consistent for all the observers and at all laser wavelengths. A possible explanation may rely on the fact that an even variability in the measurements of structures with different sizes (disc, DA and RC) is associated with different absolute changes (i.e. large for the disc, small for the RC). Consequently, in the case of DA/D, given the substantial differences between disc and RC areas, the variations in disc and DA areas (the latter being obtained by subtracting the RC from the disc area) on repeated measurements tend to be in the same direction (increase or decrease), and their variability is greater than the variability of their relative ratios.

Moreover, the CVs of DA/D and the RC/D were strongly 
Table 6. Regression statistics* for individual CVs of DA/D and RC/D (Obs. 1, infrared images. Only the right eyes of paired cases (10) were considered for regression statistics, for a total of 11 cases)

\begin{tabular}{|c|c|c|c|c|}
\hline & $\mathrm{r}$ & $\mathrm{r}^{2}$ & Slope & $\mathrm{p}$ \\
\hline \multicolumn{5}{|c|}{ CV of DA/D - Intra-image reproducibility } \\
\hline Disc area & 0.48 & 0.23 & 0.014 & 0.131 \\
\hline DA area & 0.81 & 0.66 & -0.015 & 0.002 \\
\hline $\mathrm{RC}$ area & 0.86 & 0.73 & 0.01 & 0.0007 \\
\hline $\mathrm{DA} / \mathrm{D}$ & 0.86 & 0.75 & -0.03 & 0.0005 \\
\hline $\mathrm{RC} / \mathrm{D}$ & 0.86 & 0.75 & 0.03 & 0.0005 \\
\hline \multicolumn{5}{|c|}{$\mathrm{CV}$ of $\mathrm{RC} / \mathrm{D}$ - Intra-image reproducibility } \\
\hline Disc area & 0.37 & 0.13 & -0.02 & 0.26 \\
\hline DA area & 0.66 & 0.43 & 0.028 & 0.027 \\
\hline $\mathrm{RC}$ area & 0.68 & 0.46 & -0.02 & 0.02 \\
\hline $\mathrm{DA} / \mathrm{D}$ & 0.70 & 0.49 & 0.07 & 0.015 \\
\hline $\mathrm{RC} / \mathrm{D}$ & 0.70 & 0.49 & -0.07 & 0.015 \\
\hline \multicolumn{5}{|c|}{$\mathrm{CV}$ of DA/D - Inter-image reproducibility } \\
\hline Disc area & 0.35 & 0.12 & 0.01 & 0.29 \\
\hline DA area & 0.84 & 0.71 & -0.017 & 0.001 \\
\hline $\mathrm{RC}$ area & 0.82 & 0.67 & 0.013 & 0.002 \\
\hline $\mathrm{DA} / \mathrm{D}$ & 0.85 & 0.72 & -0.04 & 0.001 \\
\hline $\mathrm{RC} / \mathrm{D}$ & 0.85 & 0.72 & 0.04 & 0.001 \\
\hline \multicolumn{5}{|c|}{ CV of RC/D - Inter-image reproducibility } \\
\hline Disc area & 0.39 & 0.16 & -0.02 & 0.23 \\
\hline DA area & 0.65 & 0.42 & 0.02 & 0.031 \\
\hline $\mathrm{RC}$ area & 0.69 & 0.48 & -0.02 & 0.018 \\
\hline $\mathrm{DA} / \mathrm{D}$ & 0.70 & 0.49 & 0.07 & 0.016 \\
\hline $\mathrm{RC} / \mathrm{D}$ & 0.70 & 0.49 & -0.07 & 0.016 \\
\hline
\end{tabular}

*CVs are the dependent variables. Optic disc parameters are the independent variables. DA: dark annulus; RC: reflective center; DA/D: dark annulus area/ disc area ratio; RC/D: reflective center area/disc area ratio.

associated with the DA and the RC areas. As optic disc of normal and glaucomatous eyes may be differentiated on the basis of "rim" and "cup" areas, the above mentioned association suggests the possibility of some distinctions in evaluating normal and glaucomatous eyes.

The results of this study are different from those reported in a previous report that used only blue and green laser wavelengths (27). We found no underestimation of disc area in comparison with conventional photographic techniques, and the intra-observer CVs of the measurements of optic disc parameters were smaller than those previously reported (range 0.0050.039 vs. $0.029-0.085$ respectively) (27). Such a difference may be explained by the different methodology used in the two studies. In fact, we evaluated images focused at the lamina cribrosa, whereas Chihara et al. (27) evaluated images focused at the level of the scleral ring, and we performed the measurements directly on the digitalized video image by means of dedicated software, whereas they performed their measurements on hard copies made with a videoprinter. Measurements performed on hard copies may be negatively affected by the low
Table 7. Inter-observer variability for infrared images: standard deviation, average of the 21 cases. Coefficient of variation is reported in parenthesis. Results obtained with the other laser wavelengths are not reported in the Table as they are very similar to those obtained with infrared light.

\begin{tabular}{lll}
\hline & Intra-image & Inter-image \\
\hline Disc & $0.073(0.035)$ & $0.073(0.034)$ \\
DA & $0.071(0.047)$ & $0.071(0.045)$ \\
RC & $0.081(0.133)$ & $0.076(0.125)$ \\
DA/D & $0.022(0.050)$ & $0.022(0.047)$ \\
RC/D & $0.022(0.100)$ & $0.022(0.094)$ \\
\hline
\end{tabular}

DA: dark annulus; RC: reflective center; DA/D: dark annulus area/disc area ratio; RC/D: reflective center area/disc area ratio. SDs of Disc, DA and RC are in $\mathrm{mm}^{2}$.

quality of the images, unlike those directly performed on a video, which have a higher resolution image.

A possible limitation of this technique of imaging the optic disc may be the potential backward bowing of the lamina cribrosa, which has been observed in both experimental acute hypertension $(28,29)$ and experimental, long-standing hypertension in primate eyes $(30,31)$. It has also been described in the optic nerve of an infant with uncontrolled congenital glaucoma (32) and in the later stages of adult glaucoma $(33,34)$. In our study, we verified that intraocular pressure at the time of imaging was within normal values, in order to rule out the possibility of "acute" changes in IOP, such as modification of the position of the lamina cribrosa at the time of imaging. Only prospective studies can evaluate such a possible bias in the morphometry of the optic disc at the level of the lamina cribrosa in cases with chronic progressive posterior bowing.

A second possible limitation of our study is the inclusion of only Caucasian subjects, as the technique described may well be affected by racial differences in fundus pigmentation, which may give rise to different tissue reflectivity.

Optic disc evaluation and morphometry by means of scanning laser ophthalmoscopy focused at the level of the lamina cribrosa was suitable and highly reproducible when the evaluation was repeated by well-trained observers. The use of long wavelength laser sources (red and infrared) allows optic disc evaluation to be performed in most cases with severe media opacities.

\section{R eferences}

-1. Jonas, J. B., Gusek, G. C. and Naumann, G. O. H. (1988) Optic disc, cup and neuroretinal rim size, configuration and correlations in normal eyes. Invest. Ophthalmol. Vis. Sci. 29, 1151-1158.

2. Katz, L. J. (1993) Optic disc drawings. In The Optic Nerve in Glaucoma (Eds. Varma, R., Spaeth, G. L. and Parker, K. W.). Pp. 147-158. J. B. Lippincott, Philadelphia.

3. Douglas, G. R. (1993) Optic disc drawings. In The Optic Nerve in Glaucoma (Eds. Varma, R., Spaeth, G. L. and Parker, K. W.). Pp. 185-192. J. B. Lippincott, Philadelphia. 
4. Takamoto, T. and Schwartz, B. (1993) Stereophotogrammetry. In The Optic Nerve in Glaucoma (Eds. Varma, R., Spaeth, G. L. and Parker, K. W.). Pp. 193-207. J. B. Lippincott, Philadelphia.

5. Heijl, A. (1993) Flicker analysis. In The Optic Nerve in Glaucoma (Eds. Varma, R., Spaeth, G. L. and Parker, K. W.). Pp. 169-183. J. B. Lippincott, Philadelphia.

6. Caprioli, J., Klingbeil, U., Sears, M. and Pope, B. (1986) Reproducibility of optic disc measurements with computerized analysis of stereoscopic video images. Arch. Ophthalmol. 104, 1035-1039.

7. Kruse, F. E., Burk, R. O. W., Volcher, H. E., Zinser, G. and Harbarth, U. (1989) Reproducibility of the optic nerve head with laser tomographic scanning. Ophthalmology, 96, 13201324.

8. Weinreb, R. N., Dreher, A. W. and Bille, J. (1989) Quantitative assessment of the optic nerve head with the laser tomographic scanner. Int. Ophthalmol. 13, 25-29.

9. Dreher, A. W. and Weinreb, R. N. (1991) Accuracy of topographic measurements in a model eye with the laser tomographic scanner. Invest. Ophthalmol. Vis. Sci. 32, 2992 2996.

10. Dreher, A. W., Tso, P. C. and Weinreb, R. N. (1991) Reproducibility of topographic measurements of normal and glaucomatous optic nerve head with the laser tomographic scanner. Am. J. Ophthalmol. 111, 221-229.

11. Lusky, M., Bosem, M. E. and Weinreb, R. N. (1993) Reproducibility of optic nerve head topography measurements in eyes with undilated pupils. J. Glaucoma, 2, 104-109.

12. Mikelberg, F. S., Wijsman, K. and Schulzer, M. (1993) Reproducibility of topographic parameters obtained with the Heidelberg retina tomograph. J. Glaucoma, 2, 101-103.

13. Cioffi, G. A., Robin, A. L., Eastman, R. D., Perell, H. F., Sarfarzai, F. A. and Kelman, S. E. (1993) Confocal laser scanning ophthalmoscope. Ophthalmology, 100, 55-62.

14. Burk, R. O. W., Rohrschneider, K., Takemoto, T., Volker, H. E. and Schwartz, B. (1993) Laser scanning tomography and stereophotogrammetry in three-dimensional optic disc analysis. Graefe's Arch. Clin. Exp. Ophthalmol. 231, 193198.

15. Woon, W. H., Fitzke, F., Poinoosawmy, D., Hitchings, R. A. and Marshall, J. (1991) Imaging the human lamina cribrosa in vivo using a confocal scanning laser ophthalmoscope. (Abstract). Invest. Ophthalmol. Vis. Sci. 32 (Suppl.), 719.

16. Fitzke, F. W. and Masters, B. R. (1993) Three-dimensional visualization of confocal sections of in vivo human fundus and optic nerve. Curr. Eye Res. 12, 1015-1018.

17. Bhandari, A., Fontana, L., Fitzke, F. W. and Hitchings, R. A. (1997) Quantitative analysis of the lamina cribrosa in vivo using a scanning laser ophthalmoscope. Curr. Eye Res. 16, 1-8.

18. Fontana, L., Bhandari, A., Fitzke, F. W. and Hitchings, R. A. (1995) Visual fields and in vivo morphometry of lamina cribrosa pores in glaucoma. (Abstract). Invest. Ophthalmol. Vis. Sci. 36 (Suppl.), 79.

19. Webb, R. H., Hughes, G. W. and Delori, F. C. (1987) Con- focal scanning laser ophthalmoscope. Appl. Opt. 26, 1492-1499.

20. Woon, W. H., Fitzke, F. W., Chester, G. H., Greenwood, D. G. and Marshall, J. (1990) The scanning laser ophthalmoscope. J. Ophth. Photogr. 12, 17-23.

21. Littmann, H. (1988) Zur Bestimmung der wahren Grosse eines Objektes auf dem Hintergrund des lebenden Auges. Klin. Monastbl. Augenheilk, 192, 66-67.

22. Brigatti, L., Bottoni, F., Miglior, S. and Orzalesi, N. (1991) Technical procedures and software for magnificationcorrected morphometry of optic disc photography. Ophthalmologica, 202, 33-37.

23. Orzalesi, N., Miglior, S., Brigatti, L. and Bottoni, F. (1991) Manual morphometry in normal and glaucomatous eyes. In Glaucoma: Concepts in Evolution (Eds. Bonomi, L. and Orzalesi, N.). Pp. 51-69. Kugler Publications, Amsterdam.

24. Miglior, S., Brigatti, L., Velati, P., Balestreri, C., Rossetti, L., Bujtar, E. and Orzalesi, N. (1994) Relationship between morphometric optic disc parameters, sex and axial length. Curr. Eye Res. 13, 119-124.

-25. Miglior, S., Brigatti, L., Lonati, C., Rossetti, L., Pierrottet, C. and Orzalesi, N. (1996) Correlation between the progression of optic disc and visual field changes in glaucoma. Curr. Eye Res. 15, 145-149.

26. Colton, T. (1979) Statistics in Medicine. Little, Brown and Co., Boston.

27. Chihara, E., Takahashi, F. and Chihara, K. (1993) Assessment of optic disc topography with scanning laser ophthalmoscope. Graefe's Arch. Clin. Exp. Ophthalmol. 231, 1-6.

28. Quigley, H. A., Flower, R. W., Addicks, E. M., McLeod, D. S. (1980) The mechanism of optic nerve damage in experimental acute glaucoma. Invest. Ophthalmol. Vis. Sci. 19, 505-517.

29. Levy, N. S., Crapps, E. E. and Bonney, R. C. (1981) Displacement of the optic nerve head: response to acute intraocular pressure elevation in primate eye. Arch. Ophthalmol. 99, 2166-2174.

30. Quigley, H. A. and Addicks, E. M. (1980) Chronic experimental glaucoma in primates: II. effect of extended intraocular pressure elevation on optic nerve head and axonal transport. Invest. Ophthalmol. Vis. Sci. 19, 137-152.

31. Radius, R. L. and Pedersen, J. E. (1984) Laser-induced primate glaucoma. II. histology. Arch. Ophthalmol. 102, 1693-1700.

32. Hetherington, J. (1993) Optic disc drawings. In The Optic Nerve in Glaucoma (Eds. Varma, R., Spaeth, G. L. and Parker, K. W.). Pp. 83-90. J. B. Lippincott, Philadelphia.

33. Quigley, H. A., Addicks, E. M., Green, R. and Maumenee, A. E. (1981) Optic nerve damage in human glaucoma: II. the site of injury and susceptibility to damage. Arch. Ophthalmol. 99, 635-649.

34. Quigley, H. A., Hohman, R. M., Addicks, E. M., Massof, R. W. and Green, R. (1983) Morphological changes in the lamina cribrosa correlated with neural loss in open angle glaucoma. Am. J. Ophthalmol. 95, 673-691. 